\author{
S. AĞ $\breve{N}^{1,4}$ \\ A. ÇELIK-AKTAŞ ${ }^{2}$ \\ J.M. ZUO ${ }^{1}$ \\ A. DANA ${ }^{3}$ \\ A. AYD1NL1 3
}

\section{Synthesis and size differentiation of Ge nanocrystals in amorphous $\mathrm{SiO}_{2}$}

\author{
${ }^{1}$ University of Illinois, Department of Materials Science and Engineering, 1304 West Green Street, \\ Urbana, IL 61801, USA \\ ${ }^{2}$ University of Illinois, Department of Nuclear, Plasma and Radiological Engineering, \\ 103 South Goodwin, Urbana, IL 61801, USA \\ ${ }^{3}$ Bilkent University, Physics Department, 06800 Ankara, Turkey \\ ${ }^{4}$ Kırıkale University, Physics Department, 71451 Kırıkale, Turkey
}

\section{Received: 10 August 2005/Accepted: 12 November 2005} Published online: 22 December 2005 • C Springer-Verlag 2005

ABSTRACT Germanosilicate layers were grown on Si substrates by plasma enhanced chemical vapor deposition (PECVD) and annealed at different temperatures ranging from $700-1010^{\circ} \mathrm{C}$ for durations of 5 to $60 \mathrm{~min}$. Transmission electron microscopy (TEM) was used to investigate Ge nanocrystal formation in $\mathrm{SiO}_{2}$ :Ge films. High-resolution cross section TEM images, electron energy-loss spectroscopy and energy dispersive X-ray analysis (EDX) data indicate that Ge nanocrystals are present in the amorphous silicon dioxide films. These nanocrystals are formed in two spatially separated layers with average sizes of 15 and $50 \mathrm{~nm}$, respectively. EDX analysis indicates that Ge also diffuses into the Si substrate.

PACS 68.73.Lp; 61.46.Hk; 61.46.-w; 68.65.Hb; 61.82.Rx

\section{$1 \quad$ Introduction}

The charge storage property of semiconductor nanocrystals embedded in an amorphous silicon oxide matrix is currently under intense investigation due to its potential application in future non-volatile memories. As charge loss through lateral paths in nanocrystal based memory devices are suppressed by the oxide isolation between nanocrystals, these devices exhibit superior charge retention characteristics compared with conventional floating-gate memory devices [1-5]. Recently, Choi et al. demonstrated the existence of memory effect in rf sputtered Ge nanocrystal devices [6]. For memory device applications, it is also crucial to control the thickness of the $\mathrm{SiO}_{2}$ tunnel oxide underneath the nanocrystal layer, as well as the density and size of the Ge nanocrystals.

Nanocrystals that are formed by precipitation from a nonuniform concentration profile often display an average size that is a function of depth [7]. Also, germanium nanoparticles formed in a Ge doped oxide layer on a silicon substrate will generally display a variation in size and concentration with depth as a result of diffusion of $\mathrm{Ge}$ towards the $\mathrm{Si} / \mathrm{SiO}_{2}$ interface $[8,9]$. Techniques, such as co-sputtering and ion implantation are typically used to obtain embedded Ge nanocrystals, the matrix usually being $\mathrm{SiO}_{2}$ [10-13]. In this letter, we report on the formation of Ge nanocrystals, with two different aver-

Fax: +00-90-312-266-4579, E-mail: aydinli@fen.bilkent.edu.tr age sizes in two separate layers in $\mathrm{SiO}_{2}$ after single step annealing of the germanosilicate layers. Germanosilicate films deposited by PECVD have some advantages due to low temperature of deposition, excellent step coverage characteristics, a high blocking effect against moisture and alkaline ions, and relatively high dielectric constant values [14]. We used TEM to determine size and crystallization. TEM is a powerful technique for the structural and chemical investigation of a wide range of materials.

Ge nanocrystals has been obtained by ion beam synthesis in $\mathrm{SiO}_{2}$ and post annealing TEM characterization showed Ge nanocrystal formation with a mean diameter of few nm's depending on implantation dose and annealing time and temperature [15]. The Ge nanocrystal size distribution of samples, rapid thermally annealed at 800 and $1000^{\circ} \mathrm{C}$ for $300 \mathrm{~s}$, has been obtained from TEM images and the best photoluminescence (PL) response was obtained with samples that exhibit uniform nanocrystal size [16]. Rapid thermal annealing at 800 and $1000^{\circ} \mathrm{C}$ for $300 \mathrm{~s}$ resulted in uniform size distribution of Ge nanocrystals, with an average size of $6.0 \mathrm{~nm}$ at $800{ }^{\circ} \mathrm{C}$, and $20-28 \mathrm{~nm}$ with multiple twinnings close to the interface when annealed at $1000{ }^{\circ} \mathrm{C}$ [16]. In samples prepared with rf magnetron sputtering nanocrystalline Ge embedded in a $\mathrm{SiO}_{2}$ matrix was obtained and examined by X-ray photoelectron spectrometry, Raman spectrometry and high resolution TEM $[11,17]$.

The precipitation and growth of Ge nanocrystals is found to be related to thermodynamical reduction of $\mathrm{GeO}$ and the diffusion of Si atoms from the Si substrate into the glassy matrix, with an aggregation of small sized Ge nanocrystals. Ge nanocrystals were also obtained by ultrahigh vacuum chemical vapor deposition (CVD) of $\mathrm{Si}_{0.75} \mathrm{Ge}_{0.25}$ on $\mathrm{Si}$ followed by high temperature oxidation. TEM studies showed that large nonspherical Ge crystallites were formed at the substrate interface [18]. On the other hand, nanoscale heterogeneity was found by TEM observation in the distribution of Ge ions in $\mathrm{SiO}_{2}: \mathrm{GeO}_{2}$ glass preforms and fibers prepared by the vapor phase axial deposition method [19]. Formation of $\mathrm{Ge}$ nanocrystallites were also studied in other matrices such as a-SiN ${ }_{x}$ deposited by the PECVD technique and followed by an annealing treatment at $800{ }^{\circ} \mathrm{C}$. It has been found that substrate temperature is a critical parameter for the formation of Ge clusters and the diffusion limited growth model was used to explain the crystallization mechanism of this mate- 
rial [20]. Ge nanocrystal size was uniform with an average size of $20 \mathrm{~nm}$ in a single layer with no distinct size separation.

In this work, the size distribution of Ge nanocrystals in $\mathrm{SiO}_{2}$ was determined from high-resolution transmission electron microscopic (HRTEM) observations. Samples for the cross-sectional HRTEM observations were prepared by standard procedures including mechanical thinning and low temperature $(200 \mathrm{~K})$ Ar-ion milling techniques.

We found that Ge nanocrystals form in two average sizes in two spatially separated layers. The size dependent spatial separation of Ge nanocrystals has been observed.

\section{Experimental procedure}

The $460 \mathrm{~nm}$ thick germanosilicate film was grown in a PECVD reactor (model PlasmaLab 8510C) on Si substrates using $180 \mathrm{sccm} \mathrm{SiH}_{4}\left(2 \%\right.$ in $\left.\mathrm{N}_{2}\right), 225 \mathrm{sccm} \mathrm{NO} \mathrm{N}_{2}$ and $90 \mathrm{sccm} \mathrm{GeH}_{4}(2 \%$ in $\mathrm{He})$ as precursor gases, at a sample temperature of $350{ }^{\circ} \mathrm{C}$, a process pressure of $1000 \mathrm{mTorr}$ under and an applied rf power of $10 \mathrm{~W}$. The composition of the grown film was determined as $\mathrm{Si}_{1.0} \mathrm{Ge}_{0.54} \mathrm{O}_{3.4}$ from $\mathrm{X}$-ray photoelectron spectroscopy measurements. Pieces cut from the same sample were annealed in nitrogen atmosphere in a quartz oven at different temperatures ranging from $700-1010^{\circ} \mathrm{C}$ for durations of 5 to $60 \mathrm{~min}$. During annealing, the samples were loaded and unloaded slowly, resulting in ramp times of $1 \mathrm{~min}$. The film was grown on $p$-type silicon substrates with resistivity of $55 \Omega \mathrm{cm}$.

Samples were prepared in cross-section orientation, so that the film layers were viewed edge-on. This preserves the information about the position of the nanocrystals with respect to the surface. Samples were then glued onto a $\mathrm{Cu}$ grid using M-Bond $600 / 610$ and the glue was cured at $150^{\circ} \mathrm{C}$ for 2 hours. Both sides of the samples were polished and mechanically ground down to $20 \mu \mathrm{m}$. To obtain samples of the right thickness for TEM observations, an ion $\mathrm{Ar}^{+}$beam of $5 \mathrm{kV}$ and incidence angle of $9-12^{\circ}$ was used. The acceleration voltage of the beam was lowered down to $1 \mathrm{kV}$ during the final stages of the thinning process in order to further minimize the $\mathrm{Ar}^{+}$induced impact damage in the area of interest. The structural characterization was carried out with a JOEL 2010F field-emission transmission electron microscope operated at $200 \mathrm{kV}$.

\section{Results and discussion}

High-resolution micrographs and selected area diffraction confirm that Ge nanocrystals are formed in our samples. As a representative example, Fig. 1 shows the dark field STEM image of a sample annealed at $1010^{\circ} \mathrm{C}$ for $1 \mathrm{~h}$. It can clearly be seen that Ge nanocrystals with well-defined spherical shapes are formed. Similar results are obtained for samples annealed between $850^{\circ} \mathrm{C}$ and $1010^{\circ} \mathrm{C}$. The crystallinity of the Ge nanoclusters was identified by selected area diffraction. The sizes of the crystalline particles were determined from the TEM images. For some nanocrystals, the actual size may be larger than the apparent size in TEM micrographs, due to cross sectioning at different sections of the particles. Nanocrystal sizes are estimated to vary in the range of 5-70 nm. It can be seen from the TEM image that these nanocrystals fall into two groups. The first group is

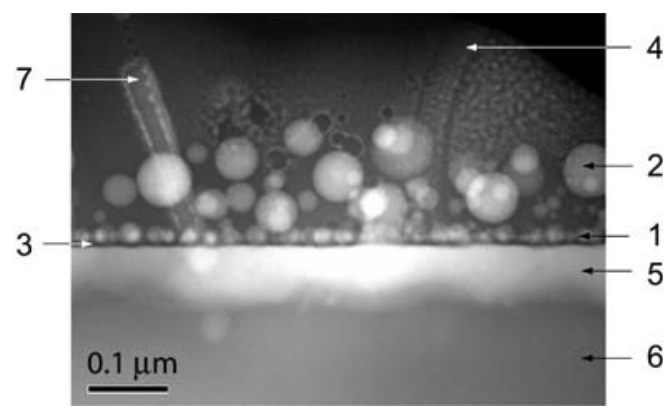

FIGURE 1 Dark field STEM image of a sample annealed at $1010^{\circ} \mathrm{C}$ for $1 \mathrm{~h}$. Ge nanocrystals are formed in the vicinity of the interface (1 and 2). Note the presence of two layers with two distinct average sizes of Ge nanocrystals. A nanocrystal free $\mathrm{SiO}_{2}$ interface oxide (3) and oxide close to the surface devoid of Ge nanocrystal formation, (4) is observed. Ge diffuses into Si substrate for an average thickness of $50 \mathrm{~nm},(5)$. Si substrate (6) and the electron damage during EDX study (7) is also indicated

composed of small nanocrystals that have an average size of $15 \mathrm{~nm}$ and a second group of large crystals that have an average size of $50 \mathrm{~nm}$. Following a $3-5 \mathrm{~nm}$ thick layer of oxide on the Si substrate, free of Ge nanocrystals, the smaller nanocrystals occupy an oxide layer of about $15 \mathrm{~nm}$. Larger nanocrystals are located in an oxide layer of $150 \mathrm{~nm}$ next to the smaller nanocrystals. The top $310 \mathrm{~nm}$ of the oxide layer is devoid of Ge nanocrystals, but not of $\mathrm{Ge}$ as observed by secondary ion mass spectroscopy (SIMS). From the TEM micrograph, a narrow band of contrast on the $\mathrm{Si}$ substrate side of the $\mathrm{Si} / \mathrm{SiO}_{2}$ interface is observed. Upon closer analysis using EDX, we find that this layer is composed of a Ge rich mixture of $\mathrm{Si}$ and $\mathrm{Ge}$. This indicates that during the $1 \mathrm{~h}$ annealing time at $1010^{\circ} \mathrm{C}, \mathrm{Ge}$ diffuses into Si substrate for an average depth of $70 \mathrm{~nm}$. Ge is known to be a fast diffuser in $\mathrm{SiO}_{2}$ beyond $800^{\circ} \mathrm{C}$ [21] Since the annealing temperature of $1010^{\circ} \mathrm{C}$ is significantly above the bulk melting point of $\mathrm{Ge}$, rapid diffusion of $\mathrm{Ge}$ is to be expected. Diffusion of Ge may be further enhanced by built-in strain effects during high temperature annealing processes.

In Fig. 2, we present a statistical analysis of the size distribution of Ge nanocrystals. We obtained a very good modified log normal fit to the data [22]. We note the absence of nanocrystals near the interface for a narrow band of oxide layer. Small nanocrystals with a mean size of $15 \mathrm{~nm}$ are crowded into a relatively narrow band in the $15 \mathrm{~nm}$ thick oxide layer. Larger nanocrystals are found in a wider band of $150 \mathrm{~nm}$ in thickness. We did not find Ge nanocrystals or clusters beyond $150 \mathrm{~nm}$ away from the interface, suggesting that oxide close to the surface is devoid of Ge nanocrystal formation. The inset of the figure shows a summary of the number of nanocrystals as a function of their location from the oxidesubstrate interface. Clustering of Ge nanocrystals into two layers is clearly observed.

Figure 3 shows HRTEM image of a Ge nanocrystal with a size of $25 \mathrm{~nm}$ formed in the germanosilicate thin film. The micrograph shows clear lattice fringes of Ge nanocrystal. The Ge nanocrystals are spherical and single crystalline. No twinnings were observed in the nanocrystals studied. We have carried out the EDX study in the STEM mode. In this mode we have used a probe size of $1.0 \mathrm{~nm}$. This probe size is much 


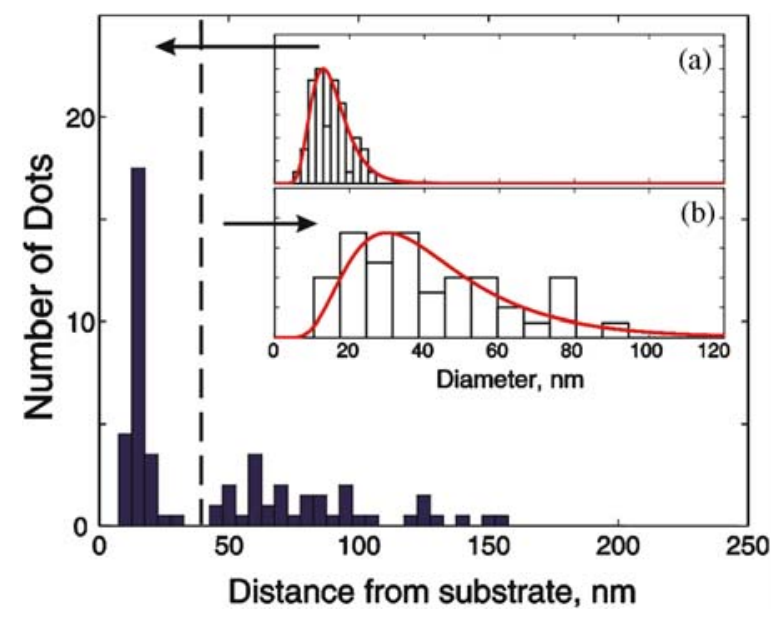

FIGURE 2 Distribution of Ge nanocrystals as a function of distance from the $\mathrm{Si}$ interface. It can be seen that a band of nanocrystals forms close to the interface, and another band forms further away from the interface. Inset: Size distributions of the two individual bands of Ge nanocrystals, (a) smaller nanocrystals are formed in the band close to the substrate, (b) larger nanocrystals form further away from the substrate

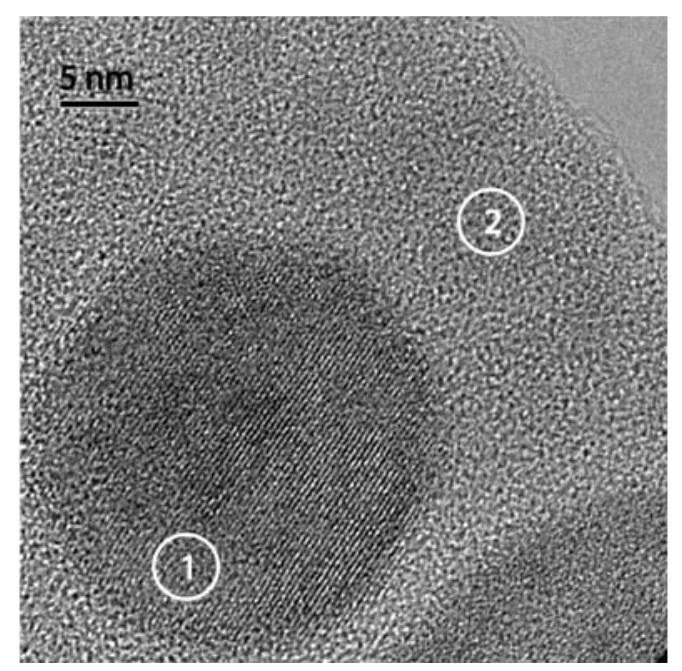

FIGURE 3 HRTEM image of a well separated Ge nanocrystal (1) with a size of $25 \mathrm{~nm}$ formed in the $\mathrm{SiO}_{2}$ matrix (2). Note the perfect alignment of crystalline planes throughout the Ge nanocrystal. No twinnings were observed

smaller than the large Ge balls in the $\mathrm{SiO}_{2}$ matrix and the $\mathrm{Ge}$ layer over the Si substrate.

From the results of the observations described above, a preliminary picture of Ge nanocrystal formation emerges. Highly mobile Ge under compressive strain [11] diffuses toward the silicon substrate forming spherical Ge nanocrystals in the oxide layer [8]. This is common to all samples annealed between 850 and $1010^{\circ} \mathrm{C}$. For samples annealed for $1 \mathrm{~h}, \mathrm{Ge}$ diffuses into the Si substrate as well. Small Ge nanocrystals form at the interface while larger Ge nanocrystals form further away from the interface. While the mechanism of formation is not clear to us, the separation of Ge nanocrystals with different sizes into two layers is a crucial result of this study. If the separation between the different sized nanocrystal bands and size distributions can be controlled and reduced to a few nanometers, such a double band formation can be especially important for flash memory applications. In a re- cent study, Ohba et al. [23] has studied the affect of double stacking of two different Si nanocrystal sizes on the retention properties of flash memory devices. They used Si nanocrystals of 5 and $10 \mathrm{~nm}$ in diameters, in two separate layers of $\mathrm{SiO}_{2}$ matrix. They found that inclusion of a double layer of nanocrystals in the oxide improves the retention time and decreases read-write voltages significantly. Our work indicates that such bilayers with small and large nanocrystals may be formed in single step annealing of PECVD grown $\mathrm{Ge}$ rich oxides. Further investigation of the dynamics of this bilayer formation may shed light into ways of controlling the size of the nanocrystals. It should also be noted that small nanocrystals at the interface are distinctly separated from the interface by a layer of $\mathrm{SiO}_{2}$ with an average thickness of $3.6 \mathrm{~nm}$, suitable for tunneling of injected carriers in a memory device.

\section{4 \\ Conclusions}

In summary, HRTEM analysis of $\mathrm{SiO}_{2}$ : Ge thin films prepared by PECVD technique has revealed the formation of Ge nanocrystals with two different sizes in the $\mathrm{SiO}_{2}$ matrix. In addition to Ge diffusing into the Si substrate for an average thickness of $70 \mathrm{~nm}$, large Ge nanocrystals with an average size of $50 \mathrm{~nm}$, form in a $150 \mathrm{~nm}$ thick layer above the lower layer with smaller Ge nanoballs, with an average size of $15 \mathrm{~nm}$, in a layer that is $15 \mathrm{~nm}$ thick. These small nanocrystals are separated from the $\mathrm{Si}$ substrate by a $3.6 \mathrm{~nm}$ thick layer of oxide. This self organized stacking of Ge nanocrystals into two size in two layers separated by an oxide layer from the Si substrate is observed for the first time and promises to be a candidate for improved flash memory applications.

ACKNOWLEDGEMENTS This work is a supported by SEMINANO, a European Union FP6 project and by TUBITAK (Turkish Scientific and Technical Research Council) through contract TBAG-U/85. Work on electron microscopy characterization was carried out at the Center for Microanalysis of Materials, University of Illinois, which is partially supported by the U.S. Department of Energy under grant DEFG02-91-ER45439. One of us (S.A.) gratefully acknowledges the financial support of the Scientific and Technical Research Council of Turkey (TUBITAK) to enable the visit to UIUC. We thank Prof. S. Suzer of Bilkent University, Chemistry Department for the XPS measurements.

\section{REFERENCES}

1 U. Serincan, G. Kartopu, A. Guenness, T.G. Finstad, R. Turan, Y. Ekinci, S.C. Bayliss, Semicond. Sci. Technol. 19, 247 (2004)

2 T. Baron, B. Pelissier, L. Perniola, F. Mazen, J.M. Hartmann, G. Rolland, Appl. Phys. Lett. 83, 1444 (2003)

3 S. Tiwari, F. Rana, K. Chan, H. Hanafi, W. Chen, D. Buchanan, Tech. Dig.Int. Electron Devices Meet. 1995, 521 (1995)

4 K. Yano, T. Ishii, T. Hashimoto, T. Kobayashi, F. Murai, K. Seki, IEEE Trans. Electron Devices 41, 1628 (1994)

5 M.L. Ostraat, J. De Blauwe, M.L. Green, L.D. Bell, M.L. Brongersma, J. Caperson, R.C. Flagan, H. Atwater, Appl. Phys. Lett. 79, 433 (2001)

6 W.K. Choi, W.K. Chim, C.L. Heng, L.W. Teo, V. Ho, V. Ng, D.A. Antoniadis, E.A. Fitzgerald, Appl. Phys. Lett. 80, 2014 (2002)

7 M. Yamamoto, T. Koshikawa, T. Yasue, H. Harima K. Kajiyama, Thin Solid Films 369, 100 (2000)

8 K.H. Heining, B. Schmidt, A. Markwitz, R. Grotzschel, M. Strobel, S. Oswald, Nucl. Instrum. Meth. B 148, 969 (1999)

9 W.K. Choi, V. Ho, V. Ng, Y.W. Ho, S.P. Ng, W.K. Chim, Appl. Phys. Lett. 86, 143114 (2005)

10 A.V. Kolobov, S.Q. Wei, W.S. Yan, H. Oyanagi, Y. Maeda, K. Tanaka, Phys. Rev. B 67, 195314 (2003) 
11 M. Zacharias, J. Blasing, J. Christen, P. Veit, B. Dietrich, D. Bimberg, Superlattices Microstruct. 18, 139 (1995)

12 V. Craciun, I.W. Boyd, A.H. Reader, E.W. Vandenhoudt, Appl. Phys. Lett. 65, 3233 (1994)

13 K.L. Teo, S.H. Kwok, P.Y. Yu, S. Guha, Phys. Rev. B 62, 1584 (2000)

14 M. Maeda, E. Yamamoto, S. Ohfuji, M. Itsumi, J. Vac. Sci. Technol. B 17, 201 (1999)

15 Q. Xu, I.D. Sharp, C.Y. Liano, D.O. Yi, J.W. Ager III, J.W. Beeman, Z. Liliental-Weber, K.M. Yu, D. Zakharov, D.C. Charzan, E.E. Haller, Mat. Res. Soc. Symp. Proc. 818, M11.17.1 (2004)

16 W.K. Choi, Y.W. Ho, S.P. Ng, V. Ng, J. Appl. Phys. 89, 2168 (2001)

17 Y. Maeda, Phys. Rev. B 51, 1658 (1995)
18 G. Taraschi, S. Saini, W.W. Fan, L.C. Kimerling, E.A. Fitzgerald, J. Appl. Phys. 93, 9988 (2003)

19 H. Hosono, K. Kawamura, H. Kawazoe, J. Nishi, J. Appl. Phys. 80, 3115 (1996)

20 X.X. Qu, K.J. Chen, M.X. Wang, Z.F. Li, W.H. Shi, D. Feng, Solid State Comm. 90, 549 (1994)

21 H. Fukuda, T. Kobayashi, T. Endoh, S. Nomura, A. Sakai, Y. Ueda, Appl. Surf. Sci. 130-132, 776 (1998)

22 L.B. Kiss, J. Soderlund, G.A. Niklasson, C.G. Granqvist, Nanostruct. Mater. 12, 327 (1999)

23 R. Ohba, N. Sugiyama, K. Uchida, IEEE Trans. Electron Devices 49, 1392 (2002) 